\title{
Structure and Spectroscopy of Transcurium Nuclei
}

\author{
I. Ahmad* \\ Physics Division, Argonne National Laboratory, 9700 South Cass Avenue, Argonne, Illinois 60439, USA
}

\author{
Received: November 13, 2001; In Final Form: November 13, 2001
}

The stability of the superheavy elements depends on the shell corrections which are governed by the single-particle spectra. Ideally one would like to experimentally determine the single-particle levels in the superheavy nuclei but the production of only a few atoms of these nuclides precludes such measurements. One therefore has to identify single-particle levels in the heaviest nuclei which are available in at least nanoCurie amounts. We have studied the structure of such heavy nuclei in the $Z=98$ region and identified many single-particle states. In particular, we have studied the structure of ${ }^{251} \mathrm{Cf}$ and ${ }^{249} \mathrm{Bk}$ by measuring the radiations emitted in the $\alpha$ decay of ${ }^{255} \mathrm{Fm}$ and ${ }^{253}$ Es. These single-particle spectra can be used to test theoretical models for superheavy elements.

\section{Introduction}

One of the fascinating areas of physics which is currently of high interest to nuclear physicists is the production and stability of superheavy elements. Quite significant progress has been made in recent years in the production of new elements. Element 112 was discovered at GSI in $1996^{1}$ and the discoveries of elements 114 and 116 were recently reported by the Dubna group. $^{2-4}$ On the theoretical side, there is some controversy about the location of the next proton shell after the $Z=82$ shell. The early calculations ${ }^{5-9}$ predicted 114 as the proton number and 184 as the neutron number for the maximum stability of superheavy elements which is confirmed by more recent Strutinsky ${ }^{10}$ type calculations. ${ }^{11-13}$ On the other hand, Relativistic Mean Field calculations ${ }^{14}$ predict the next proton shell at $Z=120$ and the Hartree-Fock self-consistent calculations ${ }^{15}$ indicate the next proton shell at $Z=126$. Clearly experimental data are needed to test the validity of these calculations.

The best way to determine the single-particle spectra in transfermium nuclei is to measure radiations emitted in their decay. These studies are, at present, not possible because of the production of only a few atoms of these nuclides. The states which are of interest in transfermium nuclei occur as excited states in the lower- $Z$ nuclei. Because of high excitation energies, these levels receive very little population in the $\alpha$ decay. Consequently large amounts of activity is needed to investigate these levels. Transcurium nuclides are produced in the High Flux Isotope Reactor (HFIR) at Oak Ridge. The heaviest nuclides produced in milliCurie quantities at HFIR are the $20-\mathrm{d}{ }^{253} \mathrm{Es}$ and $20-\mathrm{h}{ }^{255} \mathrm{Fm}$. We obtained several samples of these isotopes from Oak Ridge and studied the level structure of ${ }^{249} \mathrm{Bk}$ and ${ }^{251} \mathrm{Cf}$.

The intensity of $\alpha$ decay to an excited state in the daughter nucleus depends on the $\alpha$-decay energy $\left(Q_{\alpha}\right)$ and the formation factor which is determined by the wavefunctions of initial and final states. The dependence of ${ }^{255} \mathrm{Fm} \alpha$ intensities on $Q_{\alpha}$ is shown in Figure 1. For a $1-\mathrm{MeV}$ level in ${ }^{251} \mathrm{Cf}$, the intensity drops by five orders of magnitude due to reduction in the barrier penetration probability. The reduction due to formation factor depends on the wavefunctions of the initial and final states and typically it is in the range of 100 . Thus for a 1-MeV level, an $\alpha$ intensity of $1.0 \times 10^{-7}$ is expected. The decreased $\alpha$ intensities to the high-lying levels produce fewer $\gamma$ rays at the high energy end of the $\gamma$-ray spectrum. This means lower background due to Compton scattered photons and higher sensitivity for observing very low intensity $\gamma$ rays. Thus milliCurie sources of actinide nuclides can be used to study high-lying states in the daughter nuclei.

The formation of $\alpha$ particle in the favored decay, where the odd nucleon occupies the same state in the parent and the daugh-

*E-mail: ahmad@phy.anl.gov. FAX: +01-630-252-3612. ter nucleus, and unfavored decay is shown in Figure 2. In the favored decay, the $\alpha$ particle is formed from pairs of neutrons and protons in the same way as in the decay of an even-even nucleus. Thus favored transitions are fast and have hindrance factors of $\sim 1$. Hindrance factor is defined as the ratio of the experimental partial half-life to the half-life calculated with the spin-independent theory of Preston. ${ }^{16}$ In the unfavored $\alpha$ decay of ${ }^{255} \mathrm{Fm}$ only one pair of neutrons participate. Here, the $\alpha$ particle is formed from the unpaired neutron and one neutron from a pair. In this case the probability depends on the wavefunctions of initial and final states. The pair correlations have different effects on $\alpha$ intensities to particle states (states above the Fermi surface) and hole states (states below the Fermi surface). It has been shown by Soloviev ${ }^{17,18}$ that the $\alpha$ intensity to a level in the daughter depends on the pair occupation probability, $V^{2}$, of that level in the parent. This means that the hole states, which have higher values of $V^{2}$, have higher intensities than the particle states. For a 1-MeV state this difference corresponds to an order of magnitude. One therefore expects that hole states will have more $\alpha$ population than the particle states. In addition, phonon states exist at $\sim 1 \mathrm{MeV}$ excitation. Phonon states, in particular, $\beta$ and octupole vibrational bands, have low hindrance factors.

\section{Heavy Element Sources}

The isotopes which were used in the present study were produced in the High Flux Isotope Reactor at Oak Ridge National Laboratory as a part of the Heavy Element Production Program. Curium targets were irradiated in the reactor for about a year. The irradiated targets were processed to isolate $\mathrm{Bk}, \mathrm{Cf}$, Es, and

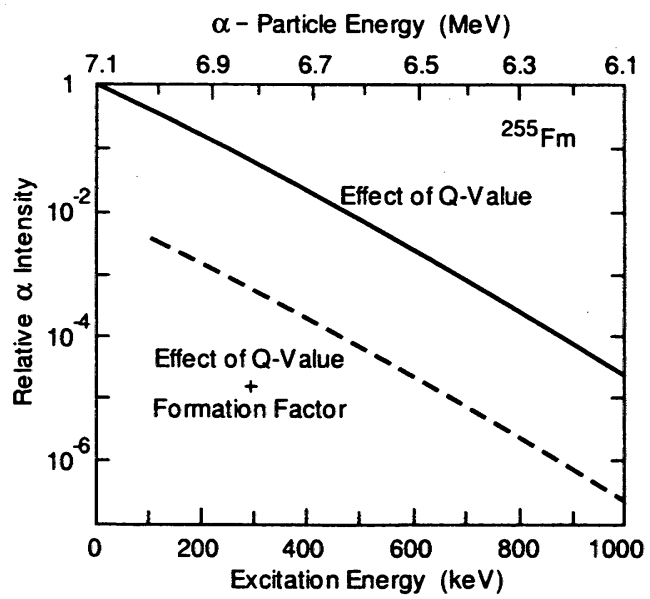

Figure 1. Dependence of ${ }^{255} \mathrm{Fm} \alpha$ intensities on the excitation energy of levels in ${ }^{251} \mathrm{Cf}$. The solid line shows the effect of $\alpha$-decay energy alone and was calculated with the spin-independent theory of Preston. ${ }^{16}$ The dashed line shows the combined effect of $Q_{\alpha}$ and formation factor. 


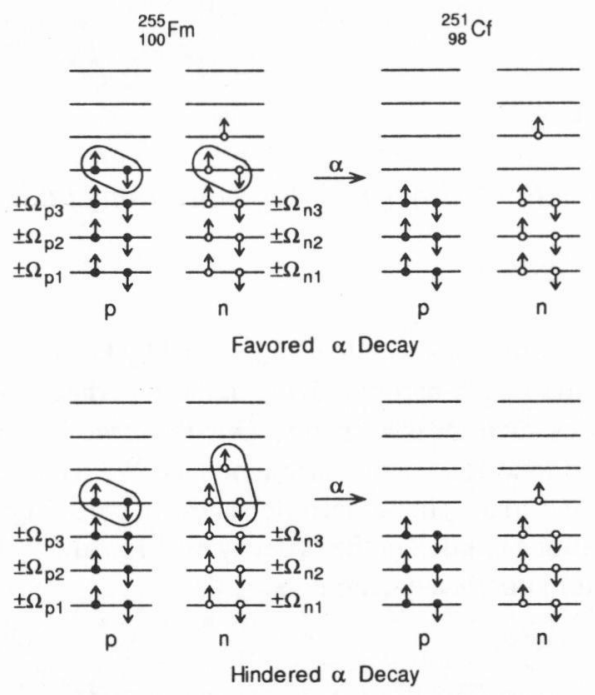

Figure 2. Effects of pair correlations on the probability of $\alpha$-particle formation. The top part shows the formation of $\alpha$ particle in favored decay, and the lower panel shows unfavored decay.

Fm. Californium and einsteinium fractions have the isotopic composition shown in Figure 3. For an isotopically pure ${ }^{253} \mathrm{Es}$ sample, Es was extracted from the Cf fraction in which ${ }^{253} \mathrm{Es}$ grew as a daughter of ${ }^{253} \mathrm{Cf}$. A chemically and isotopically pure ${ }^{255} \mathrm{Fm}$ sample was isolated from the Es fraction in which ${ }^{255} \mathrm{Es}$ decayed to ${ }^{255} \mathrm{Fm}$. Three samples of ${ }^{255} \mathrm{Fm}$, each $\sim 1 \mathrm{mCi}$, were obtained from Oak Ridge National Laboratory in December 1998 and another three in November 2000 . Two $\sim 50 \mathrm{mCi}$ samples of ${ }^{253}$ Es were acquired from Oak Ridge; one in early 1999 and the other early this year. The later samples had higher chemical and isotopic purity than the previous sources. Some samples were counted while still in the glass bottle. One Fm sample was transferred to a Pt disk in order to reduce the $\gamma$ rays due to nuclear reactions of $\alpha$ particles with light elements. Similarly, one Es sample was placed on a Ta disk to reduce background $\gamma$ rays from nuclear reactions. For $\alpha$-particle spectroscopy thin sources were prepared on glass and Pt disks.

\section{Experimental Measurements}

Except for the first ${ }^{255} \mathrm{Fm}$ sample, all samples had extremely low radioactive impurities. The later Fm samples received in November 2000 had about an order of magnitude less Es than previous samples. Gamma singles spectra of four samples were measured with a $25 \%$ coaxial Ge detector and a high-resolution $2 \mathrm{~cm}^{2} \times 7-\mathrm{mm}$ planar $\mathrm{Ge}$ detector using different absorbers to reduce the counting rates. In order to observe very weak transitions in the ${ }^{255} \mathrm{Fm} \alpha$ decay, the spectra were measured in an

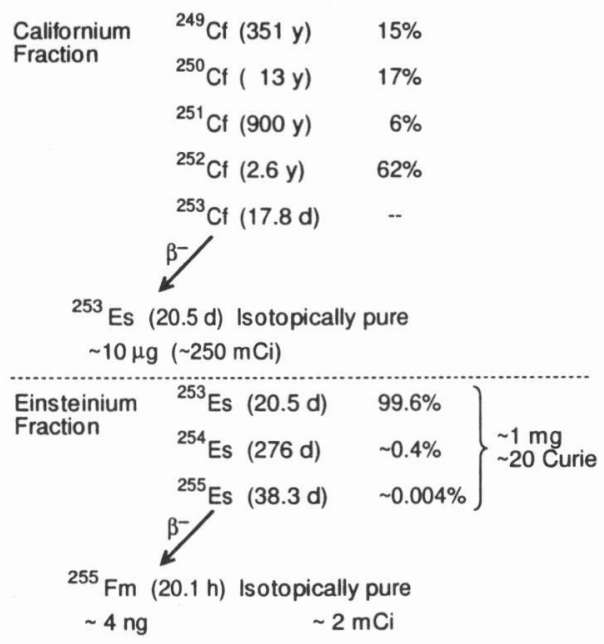

Figure 3. Isotopic composition, in atom per cent, of the $\mathrm{Cf}$ and $\mathrm{Es}$ fractions isolated from irradiated $\mathrm{Cm}$ targets at HFIR.

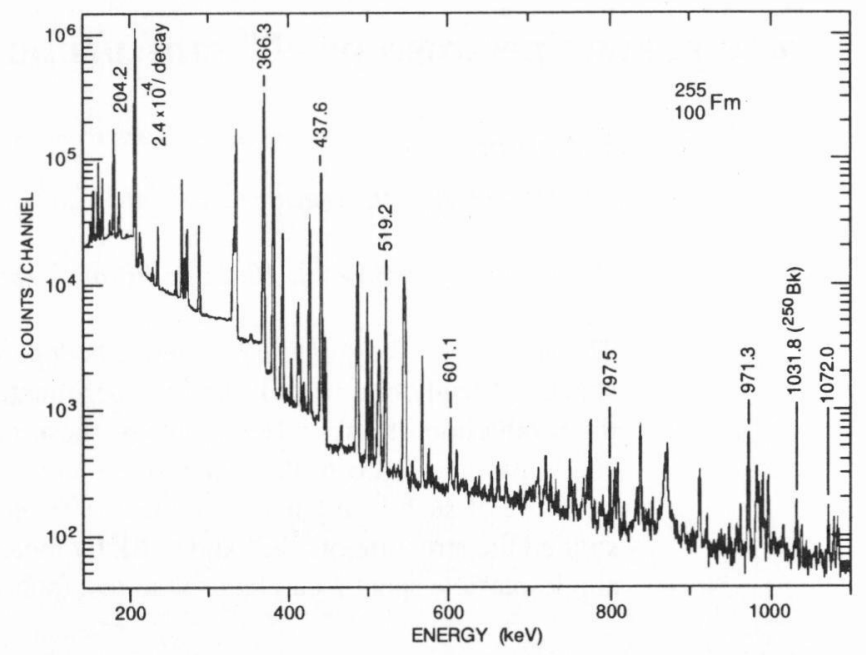

Figure 4. Gamma-ray spectrum of a ${ }^{255} \mathrm{Fm}$ sample measured with a $25 \% \mathrm{Ge}$ spectrometer. The spectrum was measured in a very low background shield for $20 \mathrm{~h}$. Most of the $\gamma$ rays identified in the spectrum have been placed in a level scheme for ${ }^{251} \mathrm{Cf}$.

extremely low background shield. Because of high resolution, the LEPS spectrum provided better information for $\gamma$ rays up to $\sim 300 \mathrm{keV}$. For higher energies, spectra measured with the $25 \%$ detector were used. These spectra contained very weak lines from Es-Bk isotopes and from reactions of $\alpha$ particles with light elements in the sample and in glass. By comparing spectra of all the four samples, we were able to identify transitions in ${ }^{255} \mathrm{Fm}$ $\alpha$ decay. A $\gamma$-ray spectrum of a sample measured in November 2000 is displayed in Figure 4. As can be seen, there is very low amount of ${ }^{254} \mathrm{Es}-{ }^{250} \mathrm{Bk}$ in the spectrum. For reference the intensity of the $971-\mathrm{keV} \gamma$ ray is $\sim 3 \times 10^{-7}$ photons per ${ }^{255} \mathrm{Fm}$ $\alpha$ decay. Gamma rays with intensities of $\sim 1 \times 10^{-8}$ photons per ${ }^{255} \mathrm{Fm} \alpha$ decay have been observed.

Gamma-gamma coincidence spectra of one ${ }^{255} \mathrm{Fm}$ sample were measured with the Gammasphere ${ }^{19}$ in December 1998 when the Gammasphere was located at Argonne National Laboratory. Most of the levels populated in the $\alpha$ decay deexcite directly to the ground state $1 / 2^{+}$band or to the $7 / 2^{+}$band at $106 \mathrm{keV}$ which decays by highly-converted low-energy transitions. These transitions were not observed in $\gamma-\gamma$ coincidence spectrum. In some cases, high-lying levels were fed by $\mathrm{K}$ con-
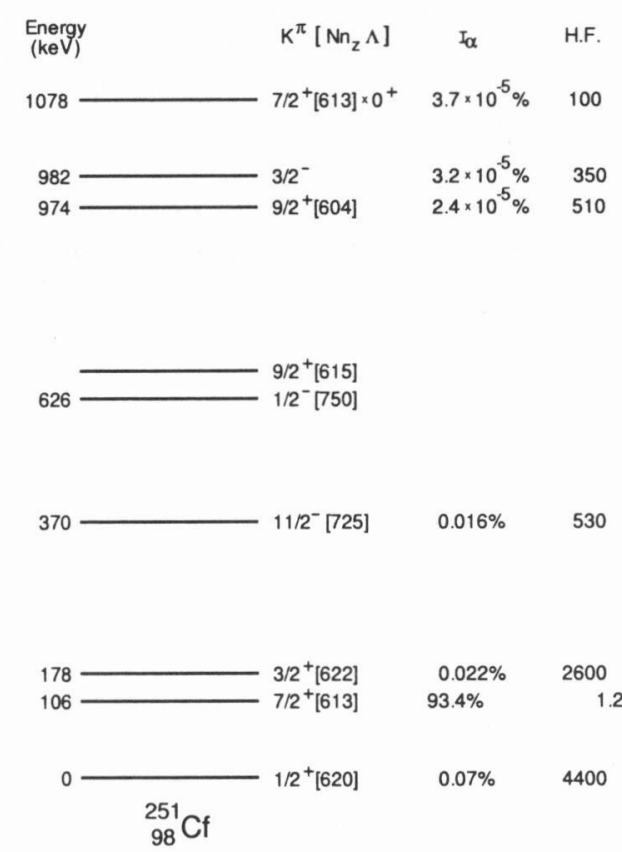

Figure 5. Intrinsic states in ${ }^{251} \mathrm{Cf}$ deduced from decay studies of ${ }^{255} \mathrm{Fm}$ and ${ }^{250} \mathrm{Cf}(d, p)$ reaction. Right hand columns represent $\alpha$ intensity in $\%$ and hindrance factor calculated with the spin-independent theory of Preston. ${ }^{16}$ 
verted transitions which allowed the observation of $\gamma$ rays deexciting to the ground band in the coincidence spectrum. Gamma rays deexciting excited states to the $3 / 2^{+}$band at $178 \mathrm{keV}$ were observed in coincidence with $\mathrm{Cf} K \mathrm{~K}$ rays because the $3 / 2^{+}$band deexcites by $\mathrm{M} 1$ transitions with large $\mathrm{K}$ conversion coefficients. The coincidence measurements allowed us to identify several new bands.

Gamma singles spectra of the two ${ }^{253}$ Es sources were measured by the same detectors used in ${ }^{255} \mathrm{Fm}$ measurements. The decay of the spectra was followed for several months. Gamma rays were assigned to ${ }^{253}$ Es $\alpha$ decay on the basis of their estimated half-life and the fact that these $\gamma$ rays are present in spectra of both Es samples. In addition, $\alpha-\gamma$ coincidence measurements were also performed using a $2 \mathrm{~cm}^{2} \mathrm{Si}$ detector and a $100 \% \mathrm{Ge}$ spectrometer. The source was $\sim 5 \mu \mathrm{Ci}$ in strength and the $\gamma$-ray spectrum was counted for one week in coincidence with $\alpha$ particles with energies less than $6.2 \mathrm{MeV}$. The spectrum showed the presence of several $\gamma$ rays including 768 - and $932-\mathrm{keV}$ peaks.

\section{Discussion}

Nuclear levels can be characterized by measurements of $\alpha$, conversion electron, and $\gamma$-ray spectra of radioactive sources and/or by charged-particle reaction spectroscopy. The former provides definite spin-parity assignments whereas the latter identifies the single-particle configuration. In an ideal case, level structure of a nucleus should be investigated by both techniques. This has been done for ${ }^{251} \mathrm{Cf}$ which provides information on neutron single-particle states and for ${ }^{251}$ Es which gives the proton single-particle spectrum.

4.1. Neutron Single-particle States. The most detailed information on the neutron single-particle states of a heavy actinide nucleus has been obtained for ${ }^{251} \mathrm{Cf}$. The level structure of this nucleus has been studied by measuring $\alpha$, electron, and $\gamma$-ray spectra of ${ }^{255} \mathrm{Fm}$, in singles and in coincidence, ${ }^{20,21}$ high

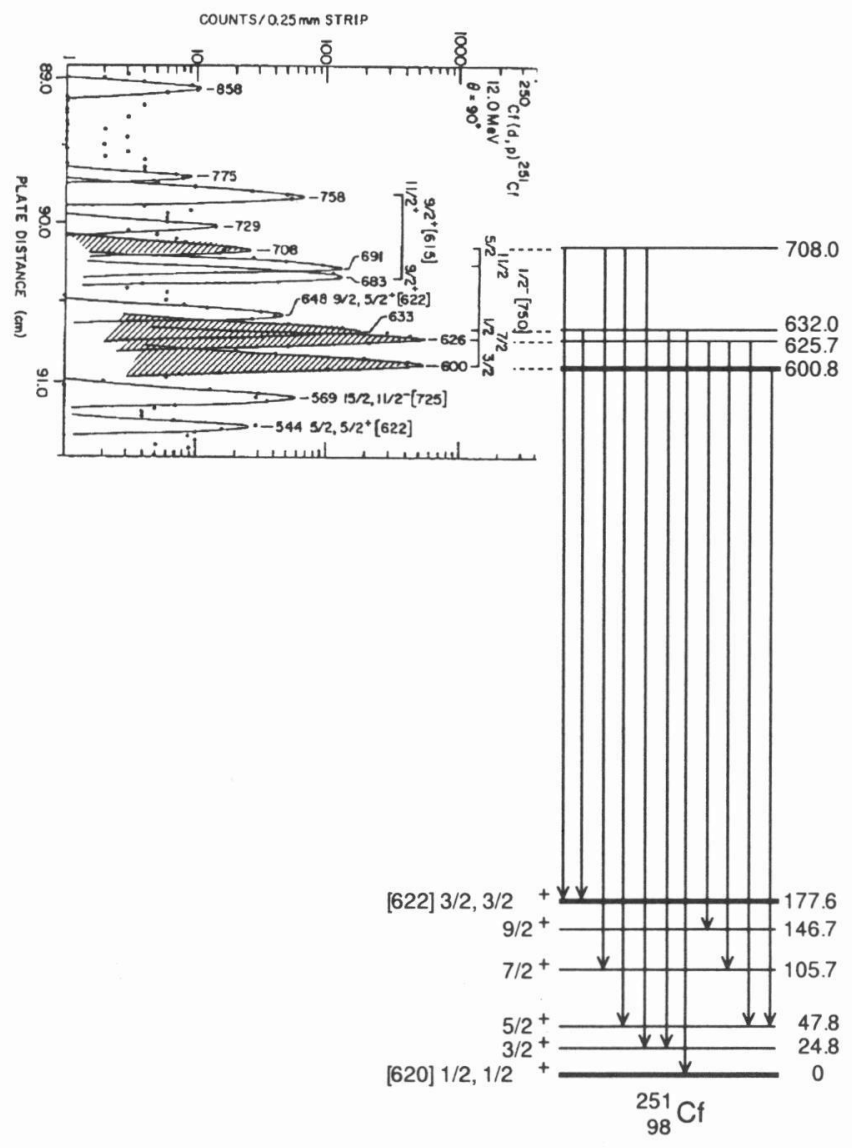

Figure 6. A portion of the ${ }^{250} \mathrm{Cf}(d, p)$ spectrum measured with an Enge split-pole magnetic spectrometer. Only peaks representing levels in the $1 / 2^{-}[750]$ band are shaded. The $\gamma$-ray transitions were observed in a $\gamma$-ray spectrum of ${ }^{255} \mathrm{Fm}$ measured with a $25 \%$ Ge spectrometer. resolution $\alpha$ spectroscopy with a magnetic spectrometer, ${ }^{22}$ and $\left.{ }^{250} \mathrm{Cf}(d, p)\right)^{251} \mathrm{Cf}$ reaction. ${ }^{23}$ The intrinsic states of ${ }^{251} \mathrm{Cf}$ obtained from these studies are shown in Figure 5. Rotational bands $1 / 2^{+}[620], 7 / 2^{+}[613], 3 / 2^{+}[622], 11 / 2^{-}[725], 9 / 2^{-}[734]$, and $5 / 2^{+}[622]$ were first observed in decay studies. The singleparticle characters of the $1 / 2^{+}[620], 7 / 2^{+}[613]$, and $3 / 2^{+}[622]$ bands were confirmed by the $(d, p)$ reaction data. In the proton spectrum from the ${ }^{250} \mathrm{Cf}(d, p)$ reaction, peaks at $633,600,708$, and $626 \mathrm{keV}$ were assigned to the members of the $1 / 2^{-}$[750] band. In our recent work, we observed $\gamma$ rays from these levels confirming their single-particle assignment. The $(d, p)$ spectrum containing the peaks from the $1 / 2^{-}$[750] band and the decay of these levels are shown in Figure 6. In our recent study, we also performed a $\gamma-\gamma$ coincidence measurement with the Gammasphere. The feeding of the members of the $1 / 2^{-}[750]$ band from higher-lying levels is shown in Figure 7, providing further evidence for spins of these states. The $1 / 2^{-}$[750] level originates from the $h_{11 / 2}$ shell state. The location of this orbital provides an estimate of the $N=184$ spherical shell. The observed levels in Figure 5 are fairly well reproduced ${ }^{21,24}$ by a single-particle model using Woods-Saxon potential.

4.2. Proton Single-particle States. Proton single-particle states have been identified in ${ }^{247} \mathrm{Bk}$ (Ref. 25,26), ${ }^{249} \mathrm{Bk}$ (Ref. $27-$ 29), ${ }^{251} \mathrm{Bk}$ (Ref. 28), and ${ }^{251}$ Es (Ref. 25, 30,31). In all these nuclei the ground state is either the $7 / 2^{+}[633]$ or the $3 / 2^{-}[521]$ orbital. The next three orbitals, $7 / 2^{-}[514], 1 / 2^{-}[521]$, and $9 / 2^{+}[624]$, have been identified in ${ }^{247} \mathrm{Bk},{ }^{249} \mathrm{Bk}$, and ${ }^{251} \mathrm{Es}$ by decay studies and $(\alpha, t)$ reactions. In ${ }^{251} \mathrm{Bk}$, only the $1 / 2^{-}[521]$ orbital, which was populated in the $\beta^{-}$decay of ${ }^{251} \mathrm{Cm}$, was identified. The best evidence for the assignments of these three orbitals was found in ${ }^{251}$ Es. The $7 / 2^{-}$[514] and the $9 / 2^{+}[624]$ states in ${ }^{251} \mathrm{Es}$ were identified in the EC decay studies of ${ }^{251} \mathrm{Fm}$ (Ref. 30) and the $\alpha$ decay of ${ }^{255} \mathrm{Md}$ (Ref. 31). The $1 / 2^{-}$[521] orbital was identified in the ${ }^{250} \mathrm{Cf}(\alpha, t)$ reaction. ${ }^{30}$

The intrinsic states of ${ }^{249} \mathrm{Bk}$ have recently been studied by measurement of $\gamma$ rays in the decay of ${ }^{253}$ Es. The low-spin states in ${ }^{249} \mathrm{Bk}$ were studied by measuring $\gamma$ rays in the decay of ${ }^{249} \mathrm{Cm}$ (Ref. 28). Levels in ${ }^{249} \mathrm{Bk}$ were also studied by ${ }^{248} \mathrm{Cm}(\alpha, t){ }^{249} \mathrm{Bk}$ reaction. However, because of poor resolution the data did not provide as good information on proton states as was achieved

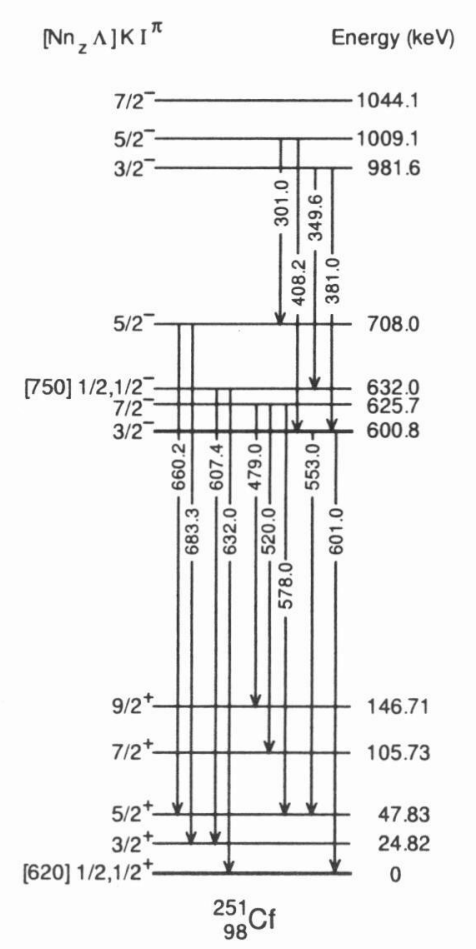

Figure 7. A partial level scheme of ${ }^{251} \mathrm{Cf}$ showing the population and decay of the $1 / 2^{-}[750]$ band. The coincidence relationship was obtained in a Gammasphere run. 


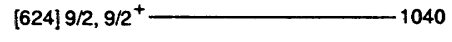

$7 / 2+[633] \times 0^{+}-932$

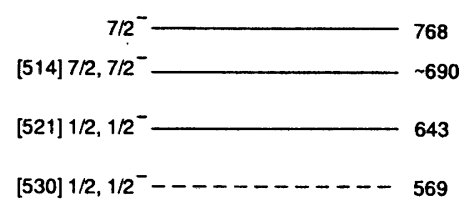

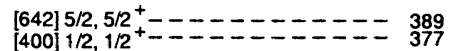

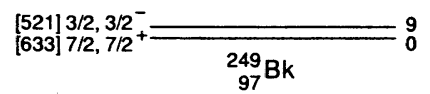

Figure 8. Intrinsic states in ${ }^{249} \mathrm{Bk}$ deduced from decay studies of ${ }^{249} \mathrm{Cm}$ and ${ }^{253} \mathrm{Es}$ and ${ }^{248} \mathrm{Cm}(\alpha, t)$ reaction. The $768 \mathrm{-keV}$ level is most likely a vibrational state.

in ${ }^{251}$ Es. Proton intrinsic states identified in all these studies are shown in Figure 8 . These levels are fairly well reproduced by single-particle model using Woods-Saxon potential. It should be pointed out that the $1 / 2^{-}$[521] orbital originates from the $f_{7 / 2}$ shell state. Thus the location of this orbital gives an estimate of the gap at $Z=114$.

\section{Summary}

Nuclear structure of nuclei around $A=250$ have been studied by a variety of techniques and many single-particle states have been identified in these nuclei. In ${ }^{251} \mathrm{Cf}$, all neutron singleparticle states between the $N=152$ and $N=162$ subshells have been identified by decay scheme studies and $(d, p)$ reaction spectroscopy. In Bk and Es nuclei, the 7/2 $[514], 1 / 2^{-}$[521], and $9 / 2^{+}[624]$ proton single-particle states, which occur above the $Z=100$, have been identified by decay scheme studies and $(\alpha, t)$ reactions. In addition, two-quasiparticle states in ${ }^{250} \mathrm{Cf}$ (Ref. 32) have also been studied. This well established nuclear structure information on nuclei near $A=250$, which are nearer to the superheavy element region than any other experimentally known region, can be used either to determine the parameters of a single-particle potential or to test the nuclear models of superheavy elements.

Acknowledgement. The author would like to thank P. Bhattacharyya, J. P. Greene, F. G. Kondev, and E. F. Moore for assistance in data analysis and R. R. Chasman for helpful discussions. This work was supported by the Department of Energy, Nuclear Physics Division, under contract No. W-31-109-ENG38. The author is also indebted for the use of ${ }^{255} \mathrm{Fm}$ and ${ }^{253} \mathrm{Es}$ to the Office of Basic Energy Sciences, U. S. Department of Energy, through the transplutonium element production facilities at the Oak Ridge National Laboratory.

\section{References}

(1)S. Hofmann, V. Ninov, F. P. Heßberger, P. Armbruster, H. Folger, G. Münzenberg, H. J. Schött, A. G. Popeko, A. V. Yeremin, S. Saro, R. Janik, and M. Leino, Z. Phys. A 354, 229 (1996).

(2) Yu. Ts. Oganessian, V. K. Utyonkov, Yu. V. Lubanov, F. Sh. Abdullin, A. N. Polyakov, I. V. Shirokovski, Yu. S.
Tsyganov, G. G. Gulbekian, S. L. Bogomolov, B. N. Gikal, A. N. Mezentsev, S. Iliev, V. G. Subbotin, A. M. Sukhov, G. V. Buklanov, K. Subotic, M. G. Itkis, K. J. Moody, J. F. Wild, N. J. Stoyer, M. A. Stoyer, and R. W. Lougheed, Phys. Rev. Lett. 83, 3154 (1999).

(3) Yu. Ts. Oganessian, A. V. Yeremin, A. G. Popeko, S. L. Bogomolov, G. V. Buklanov, M. L. Chelnokov, V. I. Chepigin, B. N. Gikal, V. A. Gorshkov, G. G. Gulbekian, M. G. Itkis, A. P. Kabachenko, A. Yu. Lavrentev, O. N. Malyshev, J. Rohac, R. N. Sagaidak, S. Hofmann, S. Saro, G. Giardina, and K. Morita, Nature 400, 242 (1999).

(4) Yu. Ts. Oganessian, V. K. Utyonkov, Yu. V. Lobanov, F. Sh. Abdullin, A. N. Polyakov, I. V. Shirokovsky, Yu. S. Tsyganov, G. G. Gulbekian, S. L. Bogomolov, B. N. Gikal, A. N. Mezentsev, S. Iliev, V. G. Subbotin, A. M. Sukhov, O. V. Ivanov, G. V. Buklanov, K. Subotic, M. G. Itkis, K. J. Moody, J. F. Wild, N. J. Stoyer, M. A. Stoyer, R. W. Lougheed, C. A. Laue, Ye. A. Karelin, and A. N. Tatarinov, Phys. Rev. C 63, 011301(R) (2001).

(5) W. D. Myers and W. J. Swiatecki, Nucl. Phys. 81, 1 (1966).

(6) H. Meldner and H. Ryde, Ark. Fys. 36, 593 (1967).

(7) S. G. Nilsson, J. R. Nix, A. Sobiczewski, Z. Szymanski, S. Wycech, C. Gustafson, and P. Möller, Nucl. Phys. A 115, 545 (1968).

(8) U. Mosel and W. Greiner, Z. Phys. 221, 261 (1969).

(9) R. R. Chasman, Proceedings of the International Symposium on Superheavy Elements, Lubbok, Texas, March 1978.

(10) V. M. Strutinsky, Nucl. Phys. A 95, 420 (1967).

(11) P. Möller and J. R. Nix, J. Phys. G 20, 1681 (1994).

(12) A. Sobiczewski, Phys. Part. Nucl. 25, 295 (1994).

(13) R. Smolańczuk, J. Skalski, and A. Sobiczewski, Phys. Rev. C 52, 1871 (1995).

(14) K. Rutz, M. Bender, T. Bürvenich, T. Schilling, P.-G. Reinhard, J. A. Maruhn, and W. Greiner, Phys. Rev. C 56, 238 (1997).

(15) S. Ćwiok, S. Dobaczewski, P.-H. Heenen, P. Magierski, and W. Nazarewicz, Nucl. Phys. A 611, 211 (1996).

(16) M. A. Preston, Phys. Rev. 71, 865 (1947).

(17) V. G. Soloviev, Phys. Lett. 1, 202 (1962).

(18) V. G. Soloviev, Theory of Complex Nuclei, translated by P. Vogel (Pergamon Press, New York, 1976).

(19) I-Yang Lee, Nucl. Phys. A 520, 641c (1990).

(20) I. Ahmad, F. T. Porter, M. S. Freedman, R. F. Barnes, R. K. Sjoblom, F. Wagner, Jr., J. Milsted, and P. R. Fields, Phys. Rev. C 1, 390 (1971).

(21) I. Ahmad, M. P. Carpenter, R. R. Chasman, J. P. Greene, R. V. F. Janssens, T. L. Khoo, F. G. Kondev, T. Lauritsen, C. J. Lister, P. Reiter, D. Seweryniak, A. Sonzogni, J. Uusitalo, and I. Wiedenhöver, Phys. Rev. C 62, 064302 (2000).

(22) I. Ahmad and J. Milsted, Nucl. Phys. A 239, 1 (1975).

(23) I. Ahmad, R. R. Chasman, A. M. Friedman, and S. W. Yates, Phys. Lett. B 251, 338 (1990).

(24) R. R. Chasman, I. Ahmad, A. M. Friedman, and J. R. Erskine, Rev. Mod. Phys. 49, 833 (1977).

(25) I. Ahmad, A. M. Friedman, R. R. Chasman, and S. W. Yates, Phys. Rev. Lett. 39, 12 (1977).

(26) I. Ahmad, S. W. Yates, R. K. Sjoblom, and A. M. Friedman, Phys. Rev. C 20, 290 (1979).

(27) J. R. Erskine, G. Kyle, R. R. Chasman, and A. M. Friedman, Phys. Rev. C 11,561 (1975).

(28) R. W. Lougheed, J. F. Wild, E. K. Hulet, R. W. Hoff, and J. H. Landrum, J. Inorg. Nucl. Chem. 40, 1865 (1978).

(29) R. W. Hoff and I. Ahmad (to be published).

(30) I. Ahmad, R. K. Sjoblom, A. M. Friedman, and S. W. Yates, Phys. Rev. C 17, 2163 (1978).

(31) I. Ahmad, R. R. Chasman, and P. R. Fields, Phys. Rev. C 61, 044301 (2000).

(32) K. Katori, A. M. Friedman, and I. Ahmad (to be published). 